\title{
ECOENDOSCOPIA NA AVALIAÇÃO PRÉ-OPERATÓRIA DO ESTÁDIO EM DOENTES COM CÂNCER GÁSTRICO: correlação com os achados cirúrgicos e/ou histopatológicos
}

\author{
Carlos Alberto MALHEIROS ${ }^{1}$, José Celso ARDENGH², Giulio Cesare SANTO³ \\ Rita de Cássia Barradas BARATA ${ }^{4}$ e Fares RAHAL $^{1}$
}

\begin{abstract}
RESUMO - Racional - O câncer gástrico é a maior causa de morte por câncer no Brasil. Na avaliação do prognóstico e no planejamento da terapêutica, é importante determinar o estádio e classificá-lo em TNM. Objetivo - Estudar os resultados da ecoendoscopia na determinação pré-operatória das categorias T e N. Métodos - Foram examinados prospectivamente e de forma consecutiva 30 pacientes com adenocarcinoma gástrico no período de 1 ano. Utilizou-se o aparelho Olympus GIF-UM-20. Após seu posicionamento na terceira porção duodenal, estudaram-se as estações linfonodais 16, 13, 12, 6, 5, 4, 3, 8, 7, 9, 10, 11, 1 e 2, usando a freqüência de 7,5 MHz. Os resultados T e $\mathrm{N}$ foram comparados com os achados cirúrgicos e/ou histopatológicos. Nos casos irressecáveis, a correlação foi feita com os achados macroscópicos. Para a análise estatística utilizou-se o teste de correlação coeficiente kappa. Resultados - Foram realizadas 16 gastrectomias totais, 7 subtotais, 5 laparotomias exploradoras e 2 videolaparoscopias. Para a categoria $\mathrm{T}$ a ecoendoscopia acertou em 25/30 casos (83,3\%). Todos os restantes 16,7\% foram superestimados. A precisão variou de $90 \%$ a $96,7 \%$, de acordo com as subdivisões T1 a T4. Para a categoria N, a ecoendoscopia foi correta em $23 / 30$ casos (76,7\%), sendo $16,6 \%$ subestimados e $6,7 \%$ superestimados. A precisão variou de $76,7 \%$ a $90 \%$, de acordo com as subdivisões N0 a N2. Conclusão - Houve nítida correlação entre a ecoendoscopia pré-operatória e os achados cirúrgicos e/ou histopatológicos em pacientes com adenocarcinoma gástrico.
\end{abstract}

DESCRITORES - Neoplasias gástricas. Adenocarcinoma. Endossonografia.

\section{INTRODUÇÃO}

O câncer do estômago é o segundo mais freqüente no Estado de São Paulo ${ }^{(27,28)}$ e a maior causa de morte por neoplasias no Brasil ${ }^{(28,38)}$. O adenocarcinoma é o tipo histológico mais freqüente, ocorrendo em $90 \%$ a $95 \%$ dos pacientes ${ }^{(7,50)}$.

Seu tratamento é essencialmente cirúrgico, já que nenhum outro método mostrou-se eficiente até o momento ${ }^{(6,13)}$. Deve ser proposto aos doentes com câncer gástrico (CG), excluindo-se os pacientes terminais e aqueles sem condições clínicas mínimas para o ato operatório. $\mathrm{O}$ objetivo da cirurgia é a cura, quando a doença é localizada e a paliação nos casos disseminados ${ }^{(49)}$.

O diagnóstico é feito pela endoscopia com biopsia em cerca de $94 \%$ dos $\operatorname{casos}^{(9)}$. Importante no pré-operatório é determinar o estádio da doença e enquadrá-la na classificação tumor, linfonodo, metástase (TNM), que avalia, além da profundidade do tumor na víscera e sua infiltração nas estruturas adjacentes, a presença de metástases linfáticas próximas ao estômago e as disseminações distantes do órgão.

Os exames de diagnóstico por imagem têm contribuído na avaliação da presença de metástases à distância (categoria M). Suas limitações dizem respeito, principalmente, ao tamanho das lesões. A ultra-sonografia (US) percutânea tem sensibilidade até $80,5 \%$ na identificação de nódulos de mais de $15 \mathrm{~mm}$ de diâmetro e apenas de $37 \%$ para os menores de $10 \mathrm{~mm}^{(52)}$. A tomografia computadorizada (TC) convencional é capaz de identificar $50 \%$ a $60 \%$ de todas as metástases hepáticas ${ }^{(45)}$, enquanto a tomografia computadorizada

Trabalho realizado no Departamento de Cirurgia da Faculdade de Ciências Médicas da Santa Casa de São Paulo e no Serviço de Endoscopia e Ecoendoscopia do Hospital Israelita Albert Einstein, São Paulo, SP

1Departamento de Cirurgia da Faculdade de Ciências Médicas da Santa Casa de São Paulo (FCMSCSP), São Paulo, SP: 2 Setor de Endoscopia da Disciplina de Anatomia e Cirurgia da Faculdade de Medicina de Ribeirão Preto, Universidade de São Paulo, Ribeirão Preto, SP; ${ }^{3}$ Departamento de Patologia da FCMSCSP; ${ }^{4}$ Departamento de Medicina Social da FCMSCSP

Correspondência: Dr. José Celso Ardengh - Al. Arapanés, 881 - cj. 111 - 04524-001 - São Paulo, SP. E-mail: jcelso@uol.com.br 
dinâmica (TCH) diagnostica até $81 \%$ delas, embora as de diâmetro inferior a $10 \mathrm{~mm}$ somente em $61 \%$ dos $\operatorname{casos}^{(22)}$.

A pesquisa pré-operatória de metástases linfonodais (categoria $\mathrm{N}$ ) mostra-se mais difícil, pela complexidade anatômica das cadeias e por identificar apenas os linfonodos aumentados de tamanho, o que nem sempre corresponde a comprometimento neoplásico ${ }^{(18)}$.

Após o diagnóstico, os esforços concentram-se na tentativa de definir a categoria $\mathrm{T}$, já que profundidade do tumor na víscera é um dos mais importantes fatores prognósticos ${ }^{(42,43)}$. A TC e a US são fiéis ao identificar lesões profundas (T3 e T4), também identificáveis pela laparoscopia. Para os casos T1 eT2, os índices de concordância desses exames com os achados histopatológicos são baixos. Nesse campo, a ecoendoscopia (EE) vem sendo utilizada em vários países, com alta precisão na classificação da categoria $\mathrm{T}^{(3,16,41)}$.

O objetivo deste trabalho foi avaliar os dados obtidos pela EE na determinação pré-operatória do estádio da doença em doentes com adenocarcinoma do estômago, verificando sua correlação com os achados cirúrgicos e/ou histopatológicos.

\section{MÉTODOS}

Foram examinados prospectivamente e de forma consecutiva 30 dos pacientes com adenocarcinoma do estômago internados no Departamento de Cirurgia da Faculdade de Ciências Médicas da Santa Casa de São Paulo entre dezembro de 1994 e fevereiro de 1996. Foram excluídos os pacientes com suboclusão ou oclusão esôfago-gástrica ou piloro-duodenal, e os em estado geral precário, incompatível com a sedação profunda necessária ao exame. O sexo, a idade, a localização primária do tumor (terço proximal, médio ou distal) foram registradas. Após a confirmação do tipo histológico pela biopsia endoscópica, foram submetidos a exames pré-operatórios de rotina na área de cirurgia do estômago e duodeno (hematológico completo, exames bioquímicos provas de coagulação, eletrocardiograma, radiografia simples de tórax e ultra-sonografia de abdome total).

\section{Ecoendoscopia}

O exame foi realizado no Serviço de Endoscopia do Hospital Israelita Albert Einstein, em São Paulo, SP, sempre pelo mesmo especialista (J.C.A.). O preparo do paciente foi iniciado com a ingestão de 30 gotas de polimetilpolixolana, seguido de anestesia tópica da orofaringe com borrifos de lidocaína a $10 \%$. Posicionamento do paciente em decúbito lateral esquerdo e monitorização com oxímetro de pulso, eletrocardiógrafo e esfigmomanômetro, com medidas de pressão arterial a cada 3 minutos e sedação profunda com propofol na dose de 2 a $4 \mathrm{mg} / \mathrm{kg}$.

Para a EE foi utilizado aparelho marca Olympus, modelo GIF UM-20, com transdutor mecânico radial rígido de $41 \mathrm{~mm}$ de extensão e 6,5 rotações por segundo, acoplado na extremidade distal. $\mathrm{O}$ transdutor permite o exame com as freqüências ultrasonográficas de 7,5 e $12 \mathrm{MHz}$, cambiáveis durante o procedimento. Passagem do aparelho sob visão direta até ultrapassar o músculo cricofaríngeo. Progressão às cegas no esôfago. Distensão da câmara gástrica, passagem para o duodeno buscando-se o posicionamento ideal, no final da segunda porção. Com movimentação em sentido cranial do aparelho, pesquisa de grupos linfonodais comprometidos nas regiões paraaórtica, da cabeça do pâncreas, retropancreática, da via biliar extra-hepática, piloro, pequena e grande curvaturas gástricas, tronco celíaco, artéria esplênica e paracárdicas, empregando sempre a freqüência de 7,5 MHz. Consideraram-se metastáticos os linfonodos hipoecogênicos, de forma arredondada e com limites nítidos. O estudo da profundidade e da extensão horizontal da lesão primária foi feito com as freqüências de 7,5 e $12 \mathrm{MHz}$. Considerou-se normal a parede gástrica com a visualização de cinco camadas ecográficas, segundo os critérios de TIO e TYTGAT $^{(48)}$, a saber: a primeira (ecogênica) seguida da segunda (hipoecogênica) correspondente à mucosa, a terceira (ecogênica) à submucosa, a quarta (hipoecogênica) à túnica muscular e a quinta (ecogênica) à serosa. As alterações desses padrões foram tidas como infiltração neoplásica.

A determinação pré-operatória do estádio obedeceu aos critérios TNM, da UICC (International Union Against Cancer) e AJCC (American Joint Committee on Cancer) e foi obtida durante o pré-operatório em todos os pacientes que tivessem boas condições operatórias.

\section{Intervenções cirúrgicas}

Todos os pacientes, que guardassem boas condições para a cirurgia, foram submetidos a uma das seguintes intervenções: videolaparoscopia, laparotomia exploradora, gastrectomia subtotal com linfadenectomia e gastrectomia total com linfadenectomia.

Nos tumores inextirpáveis, a confirmação do comprometimento de estruturas vizinhas e linfonodos, foi feita macroscopicamente durante o ato operatório, baseada no fator $\mathrm{C} 3$ da classificação TNM (evidência baseada em exploração cirúrgica) e nos achados conclusivos da JRSGC (Japanese Research Society for Gastric Cancer). Segundo essa sociedade os achados conclusivos podem ser registrados com base nos achados cirúrgicos, sem qualquer exploração histológica.

\section{Tratamento estatístico}

Compararam-se os resultados cirúrgicos e histopatológicos com os achados da EE para as categorias T e N. Verificou-se a confiabilidade da comparação com o teste de correlação coeficiente kappa. O resultado final da cirurgia ou da histopatologia foi tido como padrão-ouro. Os resultados não-concordantes foram definidos como:

- superestima: resultado falso-positivo; a EE indicou infiltração tumoral ou comprometimento linfonodal além dos constatados ao exame intra-operatório ou histopatológico.

- subestima: resultado falso-negativo; o exame mostrou invasão em profundidade ou metástase linfonodal em nível abaixo do constatado pela histopatologia ou cirurgia.

Para cada subdivisão das categorias $\mathrm{T}$ e $\mathrm{N}$ determinou-se a sensibilidade, a especificidade, o valor preditivo positivo, o valor preditivo negativo e a precisão do método.

\section{RESULTADOS}

Dos 30 pacientes analisados no presente estudo, 23 eram do sexo masculino e 7 do feminino (relação 3,3:1). A idade variou de 27 a 82 anos, com média de 63,5 anos. Quanto à localização 
primária do tumor, 9 apresentavam-no no terço proximal, 11 no médio e 10 no distal. Dois pacientes apresentavam neoplasias no coto gástrico, após gastrectomias de Billroth II. Foram realizadas 16 gastrectomias totais, 7 subtotais, 5 laparotomias exploradoras e 2 videolaparoscopias diagnósticas. Estas últimas evidenciaram infiltração de órgãos adjacentes ao estômago, metástases em fígado, linfonodos, omento, peritônio parietal e ascite.

\section{Categoria T}

A EE falhou na classificação em 5/30 (16,7\%) casos, todos superestimados: $2 \mathrm{~T} 1$ ao exame histopatológico, dados 1 como T2 e outro como T4 e 3 T3 classificados como T4. Nos demais 25/30 (83,3\%), 3 T1, 2 T2, 6 T3 e 14 T4 houve confirmação histológica (e pelos achados cirúrgicos nos T4) da classificação pela EE.

Com base nestes dados, elaborou-se a Tabela 1 de dupla entrada para os cálculos estatísticos, que mostra os resultados concordantes e conflitantes entre os achados ecoendoscópicos, cirúrgicos e histopatológicos. A análise estatística revelou coeficiente kappa $=0,7382$ com desvio padrão $=0,1151$ e $>P \cong 0$.

TABELA 1. Distribuição em dupla entrada quanto à categoria $T$, segundo os achados ecoendoscópicos, cirúrgicos e histopatológicos

\begin{tabular}{lccccc}
\hline \multirow{2}{*}{ Cirurgia/histopatologia } & \multicolumn{5}{c}{ Ecoendoscopia } \\
\cline { 2 - 6 } & T1 & T2 & T3 & T4 & Total \\
\hline T1 & 3 & 1 & & 1 & 5 \\
T2 & & 2 & & & 2 \\
T3 & & & 6 & 3 & 9 \\
T4 & & & & 14 & 14 \\
Total & 3 & 3 & 6 & 18 & 30 \\
\hline
\end{tabular}

A sensibilidade, a especificidade, o valor preditivo positivo, o valor preditivo negativo e acurácia da EE para as subdivisões da categoria T encontram-se na Tabela 2.

TABELA 2. Sensibilidade, especificidade, valor preditivo positivo, valor preditivo negativo e acurácia da USE nas subdivisões da categoria $\mathrm{T}$

\begin{tabular}{lccccc}
\hline Categoria & S $(\%)$ & E $(\%)$ & VPP $(\%)$ & VPN $(\%)$ & P $(\%)$ \\
\hline T 1 & 60,0 & 100,0 & 100,0 & 92,6 & 93,3 \\
T 2 & 100,0 & 96,4 & 66,7 & 100,0 & 96,7 \\
T 3 & 60,0 & 95,8 & 85,7 & 84,0 & 90,0 \\
T 4 & 100,0 & 81,2 & 82,3 & 100,0 & 90,0 \\
\hline S = sensibilidade; E = especificidade; VPP = valor preditivo positivo; VPN = valor preditivo negativo; P = precisão
\end{tabular}

\section{Categoria $\mathrm{N}$}

A EE errou na avaliação de 7/30 (23,3\%) dos casos. Dois classificados como N0 foram confirmados pela histopatologia como N1 (subestima). Dois N1 tratavam-se na realidade de N0 (superestima). Outros três N1 eram de fato N2 (subestima). Nos restantes 23/30 (76,7\%) casos, 7 N0, 9 N1 e 7 N2, houve confirmação pela cirurgia ou pela histopatologia da classificação pela EE. Com base nestes resultados, elaborou-se a Tabela 3 de dupla entrada para os cálculos estatísticos, que demonstra os resultados comparativos entre a ecoendoscopia, cirurgia e histopatologia. A análise estatística revelou coeficiente kappa $=0,6471$ com desvio padrão $=0,1275$ e $P \cong 0$.

A sensibilidade, a especificidade, o valor preditivo positivo, o valor preditivo negativo e acurácia da EE na avaliação das subdivisões da categoria $\mathrm{N}$ encontram-se na Tabela 4.
TABELA 3. Distribuição em dupla entrada quanto à categoria $\mathrm{N}$, segundo os achados ecoendoscópicos, cirúrgicos e histopatológicos

\begin{tabular}{lcccc}
\hline \multirow{2}{*}{ Cirurgia/ Histopatologia } & \multicolumn{4}{c}{ Ecoendoscopia } \\
\cline { 2 - 5 } & N0 & N1 & N2 & Total \\
\hline N0 & 7 & 2 & & 9 \\
N1 & 2 & 9 & & 11 \\
N2 & & 3 & 7 & 10 \\
Total & 9 & 14 & 7 & 30 \\
\hline
\end{tabular}

TABELA 4. Sensibilidade, especificidade, valor preditivo positivo, valor preditivo negativo e acurácia da USE nas subdivisões da categoria $\mathrm{N}$

\begin{tabular}{lccccc}
\hline Categoria & S (\%) & E (\%) & VPP (\%) & VPN $(\%)$ & P (\%) \\
\hline N 0 & 77,8 & 90,5 & 77,8 & 90,5 & 86,7 \\
N 1 & 81,8 & 73,7 & 64,3 & 75,0 & 76,7 \\
N 2 & 70,0 & 100,0 & 100,0 & 87,0 & 90,0 \\
\hline
\end{tabular}

$\mathrm{S}=$ sensibilidade; $\mathrm{E}=$ especificidade; $\mathrm{VPP}=$ valor preditivo positivo; $\mathrm{VPN}=$ valor preditivo negativo; $\mathrm{P}=$ precisão

\section{DISCUSSÃO}

A profundidade do tumor nas paredes do estômago é um dos principais fatores prognósticos no paciente com carcinoma gástrico $^{(4,21,30)}$, daí a importância em defini-la no pré-operatório. A TC e a US podem visualizar o espessamento da parede gástrica e a infiltração de órgãos adjacentes, porém são pouco precisos para determinar a profundidade do tumor na parede do estômago. Além disso, as lesões T1 e T2 podem passar despercebidas nestes exames, principalmente considerandose apenas o espessamento da parede gástrica ${ }^{(10)}$. A US intraoperatória da peça cirúrgica ressecada, por outro lado, atinge $93,5 \%$ de concordância com os achados histopatológicos ${ }^{(23)}$.

$\mathrm{Na}$ avaliação da categoria $\mathrm{T}$, nesta casuística, a EE concordou em 83,3\% dos casos com a histopatologia e/ou cirurgia, concordante com os dados de literatura, variáveis de $71 \%$ a $92 \%(1,16,29,41,47,48)$. Avaliou-se para cada subdivisão desta categoria a sensibilidade, a especificidade, o valor preditivo positivo, o valor preditivo negativo e a exatidão, uma vez que se estudavam apenas casos confirmados pela histopatologia e/ou cirurgia. É importante salientar que esses parâmetros não devem ser valorizados para cada um e sim aplicados ao conjunto de pacientes examinados. Obteve-se nesse estudo $100 \%$ de sensibilidade para as categorias T2 e T4, isto é, a EE foi capaz, nestes estádios, de diagnosticar corretamente todos os positivos. Entretanto, como o exame tende a superestimar a profundidade, são identificados como positivos casos em número acima do real, acarretando especificidade e valor preditivo positivo baixos, enquanto o valor preditivo negativo é alto (Tabela 2). As categorias T1 e T3, ao contrário, apresentam sensibilidades mais baixas e valores preditivos positivos altos, ou seja, quando o exame é positivo para estas classificações, a confiabilidade é alta. A precisão para todas as categorias foram elevadas, demonstrando a forte correlação entre os dois métodos diagnósticos. $\mathrm{Na}$ interpretação dos casos discordantes, é importante lembrar que o falso-positivo pode decorrer de aderências inflamatórias peritumorais $^{(26,51)}$ e o falso-negativo de infiltrações de vísceras ocas, especialmente o intestino grosso ${ }^{(43)}$. 
Vale ressaltar que a informação fundamental fornecida pelo exame é a determinação das profundidades intermediárias, $\mathrm{T} 2$ e T3 ${ }^{(36)}$. Mas a forma de alteração das cinco camadas da parede gástrica ao exame de EE pode trazer informações incorretas, como a confusão entre edema e tumor ${ }^{(29,46)}$. Isto ocorreu em um dos pacientes T1 deste estudo, superestimado em T2. Outro paciente T1 superestimado em T4, pois a lesão pré-pilórica associada a intenso processo inflamatório e edema periférico, impediu a progressão e o adequado posicionamento do aparelho (Tabela 1). A partir destes achados e as informações relatadas sobre os limites impostos ao exame pelas estenoses, passou-se a contra-indicar a EE nos casos em que a endoscopia revelava qualquer dificuldade de passagem do instrumento ${ }^{(19,43)}$.

Este estudo apresentou dois pacientes T2, ambos diagnosticados corretamente pelo exame. Dos nove casos T3, apenas seis foram corretamente identificados. Nos três restantes houve superestima para T4, por tratar-se de lesões de grandes dimensões com edema perilesional de origem inflamatória (Tabela 1). Diversos estudos comparando a EE a TC e a US, constatam a superioridade do primeiro na avaliação da categoria $\mathrm{T}^{(34,36)}$.

A avaliação macroscópica das margens cirúrgicas também pode ser passível de erro ${ }^{(6,11,20)}$. Esta informação pode ser conseguida pela $\mathrm{EE}^{(32)}$. Em dois dos pacientes deste estudo o exame revelou infiltração até a região justacárdica, impondo-se a gastrectomia total. Em ambos, o exame histopatológico confirmou a infiltração. Num terceiro caso, revelou comprometimento da submucosa até o esôfago torácico médio. A confirmação não foi possível, pois se tratava de caso tido como inoperável pela videolaparoscopia diagnóstica. Em síntese, a avaliação da extensão horizontal da neoplasia maligna nas paredes do estômago e eventual infiltração no esôfago, obtidas pela EE, é valiosa no planejamento correto da intervenção cirúrgica.

A determinação pré-operatória do comprometimento linfonodal é muito difícil, independentemente do método empregado. Trata-se, no entanto, de informação extremamente importante, devido ao seu valor prognóstico ${ }^{(25,42)}$. A maneira mais precisa para determinar o grau de comprometimento linfático é a linfadenectomia radical, conforme proposto pela JRSGC, em 1962.

A US não é boa para a pesquisa de linfonodos nas cadeias perigástricas, pela interposição, principalmente, das costelas e de gases do sistema digestório ${ }^{(47,48)}$. No entanto, comparando-se com métodos convencionais de diagnóstico por imagem, ainda apresenta resultados superiores ${ }^{(34,35,40)}$.

No exame das cadeias linfonodais, as alterações inflamatórias apresentam-se como padrão ultra-sonográfico homogêneo e hiperecóico em relação ao tumor principal e com limites pouco precisos, enquanto as neoplásicas mostram-se com padrão hipoecóico ou heterogêneo e com limites nítidos ${ }^{(7,17,47)}$. No entanto, é difícil identificar as estruturas normais e a presença de micrometástases. Considerando metastáticos os linfonodos hipoecogênicos, houve erro na avaliação de um dos pacientes N0 desta série, superestimado em N1 (Tabela 3). Entretanto, a precisão desta casuística, de $76,7 \%$, é concordante com a literatura que oscila de $52 \%$ a $83,1 \%{ }^{(2,3,8,24)}$. O maior número de erros ocorreu na avaliação da categoria N1 (Tabela 3). Dois pacientes na realidade $\mathrm{N} 0$ e superestimados em N1 não tiveram nenhum problema com a operação mais extensa realizada. Outros três foram subestimados já que se tratava de fato de casos N2. Este número de erros justifica o baixo valor preditivo positivo e a precisão menor do exame para a categoria N1. A dificuldade existente na avaliação da categoria N2 deve-se fundamentalmente a duas causas: à distância do linfonodo comprometido do transdutor posicionado na luz gástrica e ao seu tamanho. É relativamente fácil diagnosticar linfonodo metastático a mais de $3 \mathrm{~cm}$ do tumor principal, mas próximo à parede gástrica, o que não ocorre naquele a mais de $3 \mathrm{~cm}$ de distância do estômago. Obviamente é mais fácil visualizar linfonodos com maiores dimensões o que não significa, todavia, que estejam comprometidos, nem que os menores não estejam.

Apesar de suas limitações na avaliação pré-operatória do comprometimento linfonodal, a EE é considerada indispensável para os que propõem a extirpação endoscópica do carcinoma gástrico precoce, pelas suas informações quanto à profundidade da neoplasia ${ }^{(12,15,31,44)}$.

A classificação da doença dentre os estádios TNM pode ser obtida com combinações de métodos ${ }^{(5,45)}$. Nesse campo, abre-se cada vez mais espaço para a ultra-sonografia laparoscópica por reunir as informações macroscópicas da laparoscopia ${ }^{(33,37,39)}$ e dados semelhantes na categoria $\mathrm{T}$ e superiores na $\mathrm{N}$ aos da $\mathrm{EE}^{(14)}$.

Destarte, do ponto de vista prático, este estudo revela que por exemplo, para classificar uma lesão em T3 o exame ecoendoscópico permite o diagnóstico correto em $86 \%$ dos casos. Assim sendo, a EE até o momento tem se mostrado o melhor exame para a avaliação dessa categoria. Por outro lado, se for classificada em N1 ela estará correta em apenas $65 \%$ deles. Esse valor corrobora a assertiva de que a EE falha muito na identificação de nódulos linfáticos, não sendo confiável para tal objetivo.

Outro fato importante é que, do ponto de vista estatístico, fica difícil à análise conjunta $(\mathrm{TN})$ para avaliar as medidas de associação (sensibilidade, especificidade, valor preditivo positivo, valor preditivo negativo e precisão), isso só é possível ao se avaliarem as categorias isoladamente uma a uma. De forma prática pode-se descrever da seguinte forma:

"se faço ecoendoscopia de paciente com câncer gástrico e encontro o estádio $\mathrm{T} 3 \mathrm{~N} 1$, a chance de que ele pertença à classificação T3 é de $86 \%$ e de pertencer ao estádio N1 é de $65 \%$, segundo este estudo".

Como os resultados da EE para avaliar a categoria $\mathrm{T}$ são os melhores se comparados a outros métodos diagnósticos, ela pode ajudar a indicar ou contra-indicar de forma segura uma mucosectomia ou dissecção endoscópica, tornando elevado seu impacto nesse tipo de paciente.

De forma resumida pode-se dizer que os resultados deste trabalho permitem concluir que houve nítida correlação entre a avaliação pela EE e os achados cirúrgicos e/ou histopatológicos e que a EE contribuiu na avaliação pré-operatória das categorias $\mathrm{T}$ e $\mathrm{N}$ e, por conseqüência, na determinação do estádio da doença. 
Malheiros CA, Ardengh JC, Santo GC, Barata RCB, Rahal F. Endoscopic ultrasound in the preoperative staging of gastric cancer: correlation with the surgical and/or pathological findings. Arq Gastroenterol. 2008;45(1):22-7.

ABSTRACT - Background - Gastric adenocarcinoma is the major cause of death by cancer in Brazil. For the planning of the treatment and evaluation of the prognosis, the preoperative staging according to the TNM classification is very important. Aim - To evaluate the results of endoscopic ultrasound for the $\mathrm{T}$ and $\mathrm{N}$ categories. Methods - We examined 30 patients with gastric adenocarcinoma in the period of 1 year. We used a Olympus GIF UM-20 ${ }^{\mathrm{TM}}$. After positioning in the third portion of the duodenum, we started to evaluate the lymph node stations $16,13,12,6,5,4,3,8,7,9,10,11,1$ and 2 , using the frequency of $7.5 \mathrm{MHz}$. The depth of the tumor in the gastric wall was evaluated with the frequencies of 7.5 and $12 \mathrm{MHz}$. The results T and N were compared with the conclusive findings. In the unresectable cases, the correlation was made by the macroscopic findings. Results - We performed 16 total gastrectomies, 7 subtotal gastrectomies, 5 exploratory laparotomies and 2 laparoscopies. For T category, endoscopic ultrasound results were correct in 25/30 cases (83.3\%). All the other $16.7 \%$ were overestimated. The accuracy varied from $90 \%$ to $96.7 \%$ according to the subcategories T1 to T4. For the N category, endoscopic ultrasound results were correct in 23/30 cases (76.7\%). Sixteen point six percent were underestimated and $6.7 \%$ overestimated. The accuracy varied from $76.7 \%$ to $90 \%$, in agreement with the subcategories N1 to N3. Conclusion - There was a clear correlation between endoscopic ultrasound and the surgical and pathological findings in the evaluation of $\mathrm{T}$ and $\mathrm{N}$ categories in patients with gastric adenocarcinoma.

HEADINGS - Stomach neoplasms. Adenocarcinoma Endosonography.

\section{REFERÊNCIAS}

1. Akahoshi K, Misawa T, Fujishima H, Chijiiwa Y, Maruoka A, Ohkubo A Nawata H. Preoperative evaluation of gastric cancer by endoscopic ultrasound. Gut. 1991;32:479-82.

2. Akahoshi K, Misawa T, Fujishima H, Chijiiwa Y, Nawata H. Regional lymph node metastasis in gastric cancer: evaluation with endoscopic US. Radiology. 1992;182:559-64.

3. Ang TL, Ng TM, Fock KM, Teo EK. Accuracy of endoscopic ultrasound staging of gastric cancer in routine clinical practice in Singapore. Chin J Dig Dis. 2006;7:191-6.

4. Arveux P, Faivre J, Boutron MC, Piard F, Dusserre-Guion L, Monnet E, Hillon P Prognosis of gastric carcinoma after curative surgery. A population-based study using multivariate crude and relative survival analysis. Dig Dis Sci. 1992;37:757-63.

5. Botet JF, Lightdale CJ, Zauber AG, Gerdes H, Winawer SJ, Urmacher C, Brennan MF. Preoperative staging of gastric cancer: comparison of endoscopic US and dynamic CT. Radiology. 1991;181:426-32.

6. Bozzetti F, Bignami P, Bertario L, Fissi S, Eboli M. Surgical treatment of gastric cancer invading the oesophagus. Eur J Surg Oncol. 2000;26:810-4.

7. Buonadonna A, Lombardi D, De Paoli A, Bidoli E, Frustaci S. Adenocarcinoma of the stomach: univariate and multivariate analyses of factors associated with survival. Suppl Tumori. 2003;2:s31-4.

8. Cerizzi A, Crosta C, Botti F, Carrara A, Alloni R, Taschieri AM. [Preoperative staging of gastric carcinoma using endosonography (EUS)]. Ann Ital Chir. 1992;63:465-9; discussion 469-70.

9. Crawford JM. The gastrointestinal tract. In: Cotran RS, Kumar V, Robins SL, editors. Pathologic basis of disease. Philadelphia: WB Saunders; 1992. p. 755-829.

10. De Manzoni G, Pedrazzani C, Verlato G, Roviello F, Pasini F, Pugliese R, Cordiano, C. Comparison of old and new TNM systems for nodal staging in adenocarcinoma of the gastro-oesophageal junction. Br J Surg. 2004;91:296-303.

11. Demirci S, Cetin R, Yerdel MA, Kocaoglu H, Tunc G, Unal M. Value of high-resolution intraoperative ultrasonography in the determination of limits of horizontal tumor spread during surgery for gastric malignancy. J Surg Oncol. 1995;59:56-62.

12. Eriguchi M, Miyamoto Y, Fujii Y, Takeda Y, Osada I, Hagihara T, Yoshizaki I, Shimizu H, Akiyama N, Yanagie H. Regional lymph node metastasis of early gastric cancer. Eur J Surg. 1991;157:197-200.

13. Evans DB, Jessup JM, Colacchio T. Gastric cancer surgical practice guidelines Gastric Cancer Practice Guideline Committee. Oncology (Williston Park) 1997; 11:1067-72.

14. Feussner H, Kraemer SJ, Siewert JR. [The technique of laparoscopic ultrasound study in diagnostic laparoscopy]. Langenbecks Arch Chir. 1994;379:248-54.

15. Fujino MA, Morozumi A, Kojima Y, Nakamura T, Sato T, Ohtsuka H, Ohtaka M. Gastric carcinoma, an endoscopically curable disease. Bildgebung. 1994;61 (Suppl 1):38-40.

16. Ganpathi IS, So JB, Ho KY. Endoscopic ultrasonography for gastric cancer: does it influence treatment? Surg Endosc. 2006;20:559-62.

17. Giovannini M, Seitz JF, Thomas P, Houvenaeghel G, Delpero JR, Giudicelli R, Fuentes P. [Electronic sectorial ultrasound endoscopy in benign and malignant tumoral pathology of the stomach. Results in 30 patients]. Gastroenterol Clin Biol. 1993;17:26-32

18. Grimm H, Hamper K, Binmoeller KF, Soehendra N. Enlarged lymph nodes: malignant or not? Endoscopy. 1992;24 (Suppl 1):320-3.
19. Grimm H, Binmoeller KF, Hamper K, Koch J, Henne-Bruns D, Soehendra N. Endosonography for preoperative locoregional staging of esophageal and gastric cancer. Endoscopy. 1993;25:224-30.

20. Hallissey MT, Jewkes AJ, Dunn JA, Ward L, Fielding JW. Resection-line involvement in gastric cancer: a continuing problem. Br J Surg. 1993;80:1418-20.

21. Harrison JD, Fielding JW. Prognostic factors for gastric cancer influencing clinical practice. World J Surg. 1995;19:496-500.

22. Heiken JP, Weyman PJ, Lee JK, Balfe DM, Picus D, Brunt EM, Flye MW. Detection of focal hepatic masses: prospective evaluation with CT, delayed CT, CT during arterial portography, and MR imaging. Radiology. 1989;171:47-51.

23. HeintzA, Junginger T. [Endosonographic staging of cancers of the esophagus and stomach. Comparison with surgical and histopathologic staging]. Bildgebung. 1991;58:4-8.

24. Heintz A, Mildenberger P, Georg M, Braunstein S, Junginger T. Endoscopic ultrasonography in the diagnosis of regional lymph nodes in esophageal and gastric cancer--results of studies in vitro. Endoscopy. 1993;25:231-5.

25. Ichikura T, Tomimatsu S, Okusa Y, Uefuji K, Tamakuma S. Comparison of the prognostic significance between the number of metastatic lymph nodes and nodal stage based on their location in patients with gastric cancer. J Clin Oncol. 1993;11:1894-900.

26. Kockerling F, Reck T, Gall FP. Extended gastrectomy: who benefits? World J Surg. 1995; 19:541-5

27. Koifman S, Koifman RJ. Stomach cancer incidence in Brazil: an ecologic study with selected risk factors. Cad Saude Publica. 1997;13 (Suppl 1):85-92.

28. Koifman S, Koifman RJ. Environment and cancer in Brazil: an overview from a public health perspective. Mutat Res. 2003;544:305-11.

29. Lightdale CJ. Endoscopic ultrasonography in the diagnosis, staging and follow-up of esophageal and gastric cancer. Endoscopy. 1992;24 (Suppl 1):297-303.

30. Lim JH, Ko YT, Lee DH. Transabdominal US staging of gastric cancer. Abdom Imaging. 1994;19:527-31.

31. Maehara Y, Orita H, Okuyama T, Moriguchi S, Tsujitani S, Korenaga D, Sugimachi K Predictors of lymph node metastasis in early gastric cancer. Br J Surg. 1992;79:245-7.

32. Maruta S, Tsukamoto Y, Niwa Y, Goto H, Hase S, Yoshikane H. Endoscopic ultrasonography for assessing the horizontal extent of invasive gastric carcinoma. Am J Gastroenterol. 1993;88:555-9.

33. Mortensen MB, Fristrup CW, Ainsworth AP, Pless T, Nielsen HO, Hovendal C. Combined preoperative endoscopic and laparoscopic ultrasonography for prediction of R0 resection in upper gastrointestinal tract cancer. Br J Surg. 2006;93:720-5.

34. Nattermann C, Dancygier H. [Endosonography in diagnosis and staging of malignant tumors of the stomach. A prospective comparative study between endosonography, computerized tomography and conventional ultrasonography]. Z Gastroenterol. 1993;31:719-26.

35. Nattermann C, Galbenu-Grunwald R, Nier H, Dancygier H. [Endoscopic ultrasound in TN staging of stomach cancer. A comparison with computerized tomography and conventional ultrasound]. Z Gesamte Inn Med. 1993;48:60-4.

36. Okai T, Yamakawa O, Matsuda N, Kawakami H, Watanabe H, Satomura Y, Ohta $\mathrm{H}$, Motoo Y, Sawabu N, Ooi A. Analysis of gastric carcinoma growth by endoscopic ultrasonography. Endoscopy. 1991;23:121-5.

37. Oliveira DR, Ribeiro Junior U, Bitelman B, Polak M, Bresciani C, Gama-Rodrigues J, Laudanna AA, Pinotti HW. [The laparoscopy method in preoperative evaluation of patients with malignant stomach neoplasm]. Rev Hosp Clin Fac Med Sao Paulo $1993 ; 48: 4-7$ 
38. Pinto FG, Curi PR. [Mortality due to neoplasms in Brazil (1980/1983/1985): grouping by states, behavior and trends]. Rev Saude Publica. 1991;25:276-81.

39. Possik RA, Franco EL, Pires DR, Wohnrath DR, Ferreira EB. Sensitivity, specificity, and predictive value of laparoscopy for the staging of gastric cancer and for the detection of liver metastases. Cancer. 1986;58:1-6.

40. Potente G, Osti MF, Torriero F, Scattoni Padovan F, Maurizi Enrici R. [Computed tomography in the preoperative staging of gastric cancer]. Radiol Med (Torino). 1994;87:76-81.

41. Potrc S, Skalicky M, Ivanecz A. Does endoscopic ultrasound staging already allow individual treatment regimens in gastric cancer. Wien Klin Wochenschr. 2006;118 (Suppl 2):48-51.

42. Roder JD, Herschbach P, Henrich G, Nagel M, Bottcher K, Siewert JR. [The quality of life after total gastrectomy for stomach carcinoma. Esophagojejunal plication with pouch versus esophagojejunostomy without pouch]. Dtsch Med Wochenschr. 1992;117:241-7.

43. Rosch T, Lorenz R, Zenker K, von Wichert A, Dancygier H, Hofler H, Siewert JR, Classen M. Local staging and assessment of resectability in carcinoma of the esophagus, stomach, and duodenum by endoscopic ultrasonography. Gastrointest Endosc. 1992;38:460-7.

44. Sano T, Kobori O, Muto T. Lymph node metastasis from early gastric cancer: endoscopic resection of tumour. Br J Surg. 1992;79:241-4.

45. Sendler A, Dittler HJ, Feussner H, Nekarda H, Bollschweiler E, Fink U, Helmberger $\mathrm{H}$, Hofler H, Siewert JR. Preoperative staging of gastric cancer as precondition for multimodal treatment. World J Surg. 1995;19:501-8.
46. Smith JW, Brennan MF, Botet JF, Gerdes H, Lightdale CJ. Preoperative endoscopic ultrasound can predict the risk of recurrence after operation for gastric carcinoma. J Clin Oncol. 1993;11:2380-5.

47. Tio TL, Tytgat GN. Endoscopic ultrasonography in analysing peri-intestinal lymph node abnormality. Preliminary results of studies in vitro and in vivo. Scand J Gastroenterol Suppl. 1986;123:158-63.

48. Tio TL, Tytgat GN. Endoscopic ultrasonography of normal and pathologic upper gastrointestinal wall structure. Comparison of studies in vivo and in vitro with histology. Scand J Gastroenterol Suppl. 1986;123:27-33.

49. Tsendsuren T, Jun SM, Mian XH. Usefulness of endoscopic ultrasonography in preoperative TNM staging of gastric cancer. World J Gastroenterol. 2006;12:43-7.

50. Varadhachary G, Ajani JA Gastric cancer. Clin Adv Hematol Oncol. 2005:3:118-24

51. Yanai H, Fujimura H, Suzumi M, Matsuura S, Awaya N, Noguchi T, Karita M, Tada M, Okita K, Aibe T. Delineation of the gastric muscularis mucosae and assessment of depth of invasion of early gastric cancer using a 20-megahertz endoscopic ultrasound probe. Gastrointest Endosc. 1993;39:505-12.

52. Zocholl G, Kuhn FP, Augustin N, Thelen M. [Diagnostic value of sonography and computed tomography in liver metastases]. Rofo. 1988;148:8-14. 